\title{
PROCESSO DE TRABALHO DE GERÊNCIA: INSTRUMENTO POTENTE PARA OPERAR MUDANÇAS NAS PRÁTICAS DE SAÚDE?
}

Fracolli LA, Egry EY. Processo de trabalho de gerência: instrumento potente para operar mudanças nas práticas de saúde? Rev Latino-am Enfermagem 2001 setembro-outubro; 9(5):13-8.

Este estudo analisa o modo como o gerenciamento de serviços de saúde se articula à organização da produção de Unidades Básicas de Saúde, tomando como contexto o processo de implantação do modelo tecnoassistencial de SILOS. Procura-se nesse estudo operar com instrumentos analisadores que permitam compreender o processo de trabalho de gerência nos seus aspectos micropolíticos, correlacionando-o às questões macropolíticas que o perpassam. Conclui-se que existe a possibilidade de se identificar terrenos de potência no trabalho de gerência que podem produzir transformações nos serviços de saúde.

PALAVRAS CHAVE: administração em saúde pública, enfermagem em saúde pública

\section{THE MANAGEMENT WORK PROCESS: A POWERFUL INSTRUMENT TO PRODUCE CHANGE IN HEALTHCARE PRACTICE?}

This study analyzes how the management of healthcare services is articulated with the production organization in Basic Health Units by taking as a context the process of implementation of the SILOS technical care model. There is an attempt at operating with analytical instruments which enable the understanding of management work processes in their "micropolitical" aspects by correlating them to their "macropolitical" issues. It is concluded that the possibility of identifying areas of power in the management work exists, and they can produce transformation in healthcare services.

KEY WORDS: public health administration, public health nursing

\section{PROCESO DE TRABAJO DE GERENCIA: INSTRUMENTO POTENTE PARA GENERAR TRANSFORMACIONES EN LOS SERVICIOS DE SALUD?}

Este estudio analiza la manera como la gerencia de los servicios de salud se articula a la organización de la producción de la Unidades Básicas de Salud, tomando como contexto el proceso de implantación del modelo técnico-asistencial de los SILOS. Se busca en este estudio trabajar con instrumentos analizadores que permitan entender los procesos de trabajo de gerencia en su aspecto micropolítico, correlacionándolos con los aspectos macropolíticos que los circunda. Se concluye que existe la posibilidad de identificar terrenos potenciales para el trabajo de gerencia que pueden producir transformaciones en los servicios de salud.

PALABRAS CLAVES: administración en salud pública, enfermería en salud pública

\footnotetext{
${ }^{1}$ Professor Doutor, e-mail: lislaine@usp.br; ${ }^{2}$ Professor Titular, Departamento de Enfermagem em Saúde Coletiva da Escola de Enfermagem da Universidade de São Paulo
} 


\section{INTRODUÇÃO}

As práticas de saúde no Brasil têm se dicotomizado em ações médico-sanitárias (predominantemente de caráter preventivo) e ações de assistência médica individual (predominantemente de caráter curativo), com a supremacia destas em detrimento das primeiras ${ }^{(1)}$. Alguns estudiosos da saúde consideram que a superação dessa dualidade entre prevenção e cura, torna-se possível com 0 Movimento da Reforma Sanitária, na década de 80, e com a constituição do Sistema Único de Saúde (SUS).

No plano político-ideológico, pretendia-se que a construção do SUS fosse regulada pelo Estado, baseada nos princípios de universalidade, eqüidade e integralidade da atenção à saúde. Tais princípios para serem operacionalizados necessitariam estar acompanhados dos preceitos administrativos de regionalização, hierarquização e descentralização político-administrativa, bem como de formas democráticas de gestão e de controle social dos serviços de saúde.

Implementar a descentralização político-administrativa da saúde requerida pelo SUS pressupõe o preparo dos municípios para a assunção da responsabilidade de gerir os serviços públicos de saúde sob sua responsabilidade, com autonomia para organizar o processo de produção de ações de saúde segundo o modelo tecnoassistencial que lhes pareça mais apropriado.

Esse processo de descentralização administrativa, têm encontrado dificuldades diversas para se concretizar, tais como a falta de preparo técnico e econômico dos municípios para assumir suas funções gerenciais, o interesse das burocracias do setor público que, com a descentralização, perdem o manejo e a capacidade de influência político-clientelista sobre a saúde e a falta de políticas públicas de financiamento adequadas. Na atualidade, as políticas públicas de financiamento regem-se por critérios redistributivos e de eficiência, o que acaba deslocando e realocando recursos financeiros nos municípios mais preparados em detrimento dos menos preparados $^{(2-3)}$.

0 processo de descentralização e as reformas administrativas desencadeadas no Brasil na década de 80 trazem para o debate a questão das tecnologias gerenciais em saúde. Alguns autores consideram que os problemas relativos à oferta de serviços de saúde no Brasil derivam da baixa capacidade gerencial dos mesmos $^{(4)}$. A construção de conhecimentos que possam contribuir para o enfrentamento dos desafios propostos pelos SUS aponta para a questão de tomar o trabalho de gerência dos serviços de saúde como tema de importância.

De modo geral, os debates desenvolvidos na área da saúde sobre a temática gerencial e o conjunto de intervenções dos vários grupos sociais interessados nesse setor têm destacado a necessidade de operar mudanças no modo de trabalhar em gerência, em todos os níveis das organizações de saúde.

A maior parte desses debates faz referências às dimensões mais macroestruturais do trabalho de gerência em saúde e poucas têm sido as discussões que destacam as pretensões de mudanças, a partir da aquisição de capacidade teórica e operacional de ação sobre os aspectos micropolíticos desse processo de trabalho.

Sem querer negar a importância da dimensão macro estrutural no que toca à organização das práticas de saúde, este estudo vai tomar como privilegiada uma reflexão sobre as questões micropolíticas do processo de trabalho de gerência, na tentativa de procurar novas pistas para tratar as intenções de transformação que permeiam o setor saúde. Assim, o presente estudo tem como objetivo discutir as potencialidades e as limitações do processo de trabalho de gerência para operar mudanças nos modelos tecnoassistenciais de saúde.

O trabalho gerencial desenvolvido nas Unidades Básicas de Saúde (UBS) do município de Marília, no interior do Estado de São Paulo, é tomado como substrato empírico para essas reflexões.

\section{O MODELO TECNOASSISTENCIAL DE SILOS E AS ESTRATÉGIAS PARA SUA IMPLANTAÇÃO: O CASO DE MARÍLIA}

A área de saúde do município de Marília, na região oeste do Estado de São Paulo, na tentativa de viabilizar o SUS, passa por um contexto de transformações técnico-políticas que procuram viabilizar o modelo tecnoassistencial de Sistemas Locais de Saúde (SILOS).

O conceito de SILOS, considera como aspectos fundamentais para o desenvolvimento desse modelo: a reorganização do nível central de administração, a descentralização e a desconcentração da estrutura administrativa, o desenvolvimento da participação social, a implantação da intersetorialidade, a readequação dos mecanismos de financiamento do setor saúde e 0 desenvolvimento de um modelo de atenção que procure desenvolver a capacidade de análise da situação de saúde das populações, a estratégia de Vigilância à Saúde, a integração dos programas de prevenção e controle dos agravos, o reforço da capacidade administrativa local e a capacitação continuada da força de trabalho ${ }^{(5)}$.

Dessa forma, o desafio que estava colocado para o município, era o de substituir um modelo tecnoassistencial baseado principalmente no Pronto Atendimento pelo modelo SILOS ${ }^{(3)}$. As propostas para o enfrentamento desse desafio pelo município surgiram impulsionadas por um projeto transinstitucional, o projeto UNI-Marília. 
O Projeto UNI, pode ser conceitualizado como "um movimento estratégico que direciona mudanças no campo da educação dos profissionais de saúde" e procura para isso desenvolver a parceria entre serviço de saúde, academia e comunidade, para a construção e implementação de formas consensuadas de organização e prestação de assistência à saúde de indivíduos e comunidades. Nesse sentido, o município ancorado pelo projeto UNIMarília, optou pelo investimento no desenvolvimento técnico-político dos gerentes das Unidades Básicas de Saúde (UBS) para que estes pudessem então orquestrar as mudanças nos micro espaços de produção.

A Secretaria Municipal de Higiene e Saúde de Marília (SMHS), no sentido de construir seu processo de descentralização da saúde, desencadeou concomitantemente:

- um movimento técnico, que buscava constituir no dia-a-dia dos serviços uma prática baseada em território/área, dirigido para a identificação e intervenção em problemas (emergenciais e de longo enfrentamento), uma organização da assistência segundo o modelo programático e um investimento na capacitação para gerência das enfermeiras que tinham responsabilidades administrativas junto às UBS;

- um movimento político, que procurava as condições legais e formais para institucionalizar as mudanças técnicas e políticas que estavam sendo construídas.

O presente estudo tem como objetivo compreender "como" o processo trabalho de gerência (re)articula a organização da produção da UBS, tendo como finalidade práticas baseadas em território/área e no modelo programático citado anteriormente, e qual sua potência para a (re)construção de modelos tecnoassistenciais em saúde.

\section{BASES METODOLÓGICAS DO ESTUDO}

Por considerar que os componentes funcionais da sociedade são fenômenos e não podem ser compreendidos fora do contexto em que emergem este estudo optou pela dialética materialista como uma metodologia de investigação que possibilita a apreensão do real "em estruturação" e a construção de novas sínteses no plano do conhecimento e da realidade histórica.

$O$ processo de trabalho de gerência em UBS envolve um duplo movimento, de um lado, a execução de práticas gerenciais que tomam como seu campo de ação o território de formulação e decisão política e, de outro lado, o território de práticas gerenciais que enfrentam o fabricar "bens" do trabalho em saúde. O processo de trabalho de gerência em UBS torna-se o lugar de possíveis intervenções impactantes sobre a política e o modelo de atenção à saúde ${ }^{(6)}$

Apostando nessa potencialidade do trabalho gerencial, estabelece-se uma reflexão sobre as frentes de luta que podem ser tomadas quando se busca construir processos concretos de práticas de saúde diferenciadas no interior dos serviços de saúde, práticas estas que consigam romper com o instituído e viabilizar a construção de "novos" instituíntes ${ }^{(6)}$. Para a construção de proposições teóricas que viabilizem a construção desses novos "instituintes", recorre-se neste estudo às concepções de trabalho em saúde, enfocando sua dinâmica trabalho vivo/trabalho morto e às teorias gerenciais relativas à natureza do trabalho de gerência em serviços de saúde.

O trabalho em saúde é um processo produtor de interseções, e a análise dessas interseções, no interior dos processos de trabalho, articuladas à micropolítica instituída ou instituinte desses processos, se constitui em uma estratégia que pode revelar "ruídos" e "linhas de fuga" para o trabalho vivo operador de mudanças ${ }^{(6)}$. Além disso, os processos de trabalho se constituem em analisadores institucionais privilegiados e a análise desses processos pode revelar os diferentes projetos em disputa no interior dos equipamentos de saúde ${ }^{(6)}$.

Algumas propostas teóricas entendem que os serviços de saúde se parecem mais com "arenas de disputas" do que com "organismos vivos" (como descrito pelas teorias funcionalistas de gerência). Dessa forma, a dinâmica do trabalho nos serviços de saúde se assemelha (em muito) a um jogo de xadrez, no qual os trabalhadores (da mesma forma que os jogadores) operam segundo uma rede de petições e compromissos entre eles; compartilham significações que balizam um certo contrato de relações e não podem ser sujeitos plenos mas "bem sujeitados" às regras do jogo ${ }^{(6)}$.

Compreendido a partir dessas concepções, o objeto em estudo (o processo de trabalho de gerência) assume uma expressiva dependência de dimensões mais singulares (relativas ao sujeito que concretamente executa o trabalho de gerência), o que nos levou a buscar na Análise Institucional o conhecimento para articular conceitos da psicanálise e do materialismo histórico para conhecer e intervir nas organizações sociais.

Os dados empíricos deste estudo foram obtidos através de entrevistas com as gerentes das UBS e através de análises documentais.

Foram sujeitos deste estudo os profissionais enfermeiros que atuavam nas UBS do município de Marília, os quais eram na sua totalidade mulheres. Os dados foram coletados utilizando a técnica de grupo focal, estes foram organizados sob a forma de "fluxogramas analisadores". 0 "Fluxograma Analisador" ${ }^{(6)}$ se constitui numa 
ferramenta que interroga os para que; os que e os como do processo de trabalho, e ao mesmo tempo revela a maneira de governá-lo*.

A partir das informações obtidas com a aplicação dessa ferramenta analisadora aos depoimentos das gerentes das UBS de Marília, foi possível a construção de "Fluxogramas Analisadores" ** representativos do trabalho de gerência desenvolvido pelas enfermeiras nessas unidades de saúde.

A análise desses fluxogramas nos permite apontar neste estudo as lógicas institucionais presentes na molecularidade do processo de trabalho de gerência desenvolvido nas UBS de Marília.

\section{RESULTADOS E ANÁLISES}

Segundo os relatórios do grupo de avaliação do Projeto UNI-Marília, ao final de 1996, a estrutura organizativa da SMHS era considerada inadequada para que o município pudesse implementar a proposta de descentralização administrativa requerida pelo modelo de SILOS. A solução encontrada pela SMHS para superar essa dificuldade foi à adoção de uma estrutura organizacional e operacional que buscava romper com a centralização de poder na figura do Secretário de Saúde, através da instituição do Conselho Municipal de Saúde (COMUS) e do Fundo Municipal de Saúde como instâncias deliberativas da SMHS e a criação de coordenações regionais de saúde diretamente articuladas às UBS. Contudo, não instituía uma central de planejamento, instância importante no modelo de SILOS, uma vez que a base desse modelo tecnoassistencial é a identificação e intervenção nos problemas de saúde existentes num dado território. Essa estrutura organizacional instituída previu a existência de um Núcleo de Informação. Contudo o mesmo operava vinculado ao Setor de Faturamento, o que imprimia um caráter de controle quantitavofinanceiro de procedimentos em detrimento de ações de monitoramento dos perfis epidemiológicos regionais e locais, conforme previsto no modelo de SILOS.

A estrutura organizacional construída mantinha além desse outros problemas como: a centralização do setor administrativo (responsável pelos recursos humanos e pelo almoxarifado), a centralização da Vigilância Epidemiológica e Sanitária, e a centralização da "Saúde do Servidor", todas independentes das instâncias regionais e locais ${ }^{(7)}$.

Para operacionalizar, no âmbito político-legal, a descentralização administrativa das UBS a SMHS de Marília aprovou um decreto lei que instituiu o cargo de "Chefe de Unidade de Saúde", no sentido de dar legitimidade técnico-política à função administrativa das enfermeiras. Contudo, as prerrogativas dessa função não foram definidas a priori, abrindo com isso possibilidades e limites para a construção da prática gerencial. A descrição da experiência de implantação de reformas na Secretaria de Saúde de Betim (MG), chama a atenção para a importância de se ter claramente estabelecido o papel dos gerentes locais e suas relações com o projeto de saúde do município, para que os mesmos possam ser mais efetivos na construção de reformas necessárias ${ }^{(8)}$. Essa ausência de definições relativas às atividades dos "Chefes de Unidade de Saúde" criou uma "autonomia" gerencial que se constituiu em um núcleo de tensão para as gerentes no município de Marília.

A análise dos processos micropolíticos do trabalho de gerência revelou que, as lógicas que caracterizavam este trabalho expressavam-se através de compromissos com atividades-meio que organizavam 0 trabalho médico. Identificou-se também que estas lógicas estavam organizadas sob uma concepção clássica de gerência, isto implicava na adoção, pelas gerentes, de tecnologias e saberes gerenciais pautados em modelos tayloristas, fordistas e weberianos. Os saberes utilizados pelas gerentes reforçavam a burocracia, a divisão do trabalho, o produtivismo e a alienação do trabalhador do seu produto final, bem como enfatizavam ações de supervisão e controle e deixavam para segundo plano o planejamento e a avaliação dos profissionais e das práticas de saúde. No interior do processo de trabalho desenvolvido pelas gerentes, a tensão entre assistir e gerenciar aparecia como pólos de oposição e não como ações intercessoras e intercomplementares.

O modelo médico-liberal era a lógica predominante na organização da produção na UBS, embora a lógica do modelo de Vigilância à Saúde buscava instituir-se. Essa tensão, entre instituído e instituinte, produzia "ruídos" no trabalho de gerência, os quais se expressavam principalmente na dificuldade da gerente em articular ações intersetoriais para o enfrentamento dos problemas cotidianos da UBS.

As relações estabelecidas entre as gerentes e as instâncias centrais da SMHS indicavam que no campo do simbólico organizacional a descentralização ainda não havia ocorrido. As gerentes das UBS de Marília coletavam e organizavam as informações de saúde, contudo não realizavam a análise dessas

\footnotetext{
* Para maiores esclarecimentos sobre o uso do fluxograma analisador como instrumento de intervenção e pesquisa institucional, consultar Merhy EE. Em busca de ferramentas analisadoras das tecnologias em saúde: a micropolítica do trabalho vivo em saúde. In: Merhy EE, Onocko R. AGIR EM SAÚDE: um desafio para o público. São Paulo (SP): HUCITEC; 1997. p.71-112

** Os fluxogramas analisadores referidos neste texto como base empírica das análises apresentadas, encontram-se descritos em Fracolli LA. Processo de trabalho de gerência: possibilidades e limites frente à reorganização do trabalho na rede básica de saúde de Marília. [tese]. São Paulo (SP): Escola de Enfermagem/USP; 1999
} 
informações com vistas a identificar os problemas/necessidades de saúde de sua área e a partir disso reorganizar a produção da UBS no sentido de atender a essas necessidades/problemas identificados.

A análise micropolítica do processo de trabalho de gerência, apontou que a enfermeira, ao desempenhar um papel gerencial, o faz como uma visão idealista e disciplinadora do trabalho em saúde e "frustra-se" ao perceber que as "coisas" não acontecem como ela espera, chegando algumas vezes a tomar para si a "culpa" dos "quebras" cotidianos do processo de trabalho. Tal fato torna o trabalho gerencial uma fonte constante de tensão para a enfermeira.

\section{O TRABALHO DE GERÊNCIA EM SERVIÇOS DE SAÚDE: QUE DESAFIOS PRECISAM SER SUPERADOS?}

Os "ruídos" do trabalho de gerência apresentados neste estudo permitem algumas sínteses, as quais podem, de alguma maneira, responder a questão inicial, relativa à potência do trabalho de gerência para a transformação dos modelos de saúde.

A conformação da rede básica de serviços de saúde responde a um longo processo social que expressa as reordenações da relação entre o Estado e as classes sociais, através das políticas de saúde, e portanto as ações tecnológicas (inclusive as tecnologias gerenciais) se conformaram para viabilizar esse contexto ${ }^{(9)}$. O modelo tecnoassistencial adotado para a construção da rede básica de saúde em Marília, buscava articular a lógica clínica e epidemiológica, sob a ótica de um planejamento baseado na relação custo/eficácia ${ }^{(3)}$. Essa forma de conceber a rede básica reduz a questão da saúde e da doença a uma questão de organização racional das ações médicas, auxiliadas pelas ações coletivas, nos momentos da promoção e proteção à saúde.

O modelo tecnoassistencial de SILOS, busca superar essas características medicalizantes e procedimento-centrada do modelo anterior, contudo não abandona os princípios da visão sistêmica, no qual a base de controle seja um planejamento único de ações de saúde, centrado em uma rede pública que articula as demandas por serviços médicos, com o conjunto dos serviços de maior complexidade de retaguarda. O modelo de SILOS traz também para seu interior conceitos formados pela saúde pública no campo administrativo/ gerencial (como a regionalização, a descentralização, etc.), o que faz desse modelo uma mistura entre uma organização administrativa e programática, baseada nas ações coletivas, e uma abordagem tecnológica marcada pela ótica médica, mas sob uma base epidemiológica.
As análises realizadas apontaram que 0 trabalho de gerência é um trabalho sensivel as lógicas institucionais prédeterminadas (instituídas), e os ruídos que manifesta apontam para as contradições internas do modelo SILOS. Contudo o trabalho de gerência guarda dentro de si possibilidades instituintes. Essas possibilidades instituintes encontram-se diretamente relacionadas aos agentes desse trabalho e aos seus instrumentos de atuação. Ao tratar dos primeiros (os agentes do trabalho de gerência) aponta-se para o projeto político que a enfermagem tem para si enquanto ator social; para a maneira como coloca seu projeto para disputar no cenário da saúde e para a forma de se aliar a outros sujeitos sociais dentro desse cenário de disputas.

Em Marília, a predominância de enfermeiras nos cargos gerenciais carreia para esse trabalho uma certa homogeneidade técnica e política que não foi capaz de romper com as lógicas institucionais que tradicionalmente vêm conformando o trabalho em saúde. A ausência de uma definição clara dos limites do cargo de "Chefe de Unidade de Saúde" se constituía em um espaço possível de construção do novo, de apresentação do projeto da enfermagem para a gerência de UBS e para a instituição do modelo tecnoassistencial no qual acreditamos. Infelizmente não conseguimos transformar esse espaço potencial em espaço real e continuamos a reiterar o modelo médico-hegemônico.

A análise das tecnologias do trabalho de gerência utilizadas pelas enfermeiras, sugere que, a estas, sejam incorporados dispositivos gerenciais que possam romper com visões "tradicionais" do que seja gerenciar um serviço de saúde. Sugere-se a utilização de instrumentos gerenciais que propiciem a "auto-análise" e a "autogestão" dos trabalhadores de saúde ${ }^{(6)}$.

Com isso, observa-se que as distintas experiências que buscam a mudança efetiva do processo de trabalho em saúde, têm necessidade de incorporar as questões que surgem no âmbito micropolítico do trabalho em saúde e, para tanto, a utilização de ferramentas como o "Fluxograma Analisador" mostra-se pertinente para a identificação desses aspectos.

As relações macro e micropolíticas na saúde encontramse inscritas nos espaços de gestão do processo de trabalho e das organizações de saúde, e as configurações que estas adquirem passam necessariamente pela presença do trabalho vivo em ato. Assim, mais do que questionar o que ocorre nos serviços de saúde a partir de um modelo "a priori" de organização do processo de trabalho em saúde, devemos desenvolver a capacidade de criar interrogações sobre o que está ocorrendo, abrindo com isso possibilidades para o trabalhador coletivo "inventar" modos novos e singulares de realizar o trabalho em saúde em situações concretas ${ }^{(6)}$. 


\section{REFERÊNCIAS BIBLIOGRÁFICAS}

1. Menezes CS. A unicidade do sistema único de saúde em questão: a integralidade e a hierarquização do cuidado sob a perspectiva do trabalho vivo. [dissertação]. Campinas (SP): Faculdade de Ciências Médicas/UNICAMP; 1998.

2. Marsiglia RMG. Servidor, funcionário, trabalhador: interesses e culturas organizacionais no setor público de saúde. [tese]. São Paulo (SP): Faculdade de Filosofia, Letras e Ciências Humanas/USP; 1993.

3. Chirelli MQ. $O$ processo de municipalização dos serviços públicos de saúde em Marília/SP, 1983 a 1992. [dissertação]. Ribeirão Preto (SP): Escola de Enfermagem/USP; 1995.

4. Seixas JC. Mensagem ao leitor. Rev Esp Saúde 1996 Set; 5(5):3. 5. Silva AG. Modelos tecnoassistenciais em saúde: 0 debate no campo da saúde coletiva. [tese]. Rio de Janeiro (RJ): Escola Nacional de Saúde Pública/FIOCRUZ; 1996.
6. Merhy EE. Em busca de ferramentas analisadoras das tecnologias em saúde: a micropolítica do trabalho vivo em saúde. In: Merhy EE, Onocko R, organizadores. Agir em saúde: um desafio para o público. São Paulo (SP): Hucitec; 1997. p. 71-112.

7. Fracolli LA. Processo de trabalho de gerência: possibilidades e limites frente à reorganização da rede básica de saúde em Marília. [tese]. São Paulo (SP): Escola de Enfermagem/USP; 1999.

8. Bueno WS. Betim: Construindo um gestor pleno. In: Merhy EE, Onocko R, organizadores. Agir em saúde: um desafio para o público. São Paulo (SP): Hucitec; 1997. p. 169-96.

9. Merhy EE. A saúde pública como política: São Paulo 1920-1948, os movimentos sanitários, os modelos tecnoassistenciais e a formação das políticas governamentais. São Paulo (SP): Hucitec; 1992. 